\title{
Diagnostic Accuracy of Three-dimensional Ultrasonography in Detection of Endometrial Lesions compared with Hysteroscopy in Infertile Women
}

\author{
${ }^{1}$ Firoozeh Ahmadi, ${ }^{2}$ Hadieh Haghighi, ${ }^{3}$ Zahra Ghahremani, ${ }^{4}$ Fatemeh Niknejad, ${ }^{5}$ Farnaz Akhbari \\ ${ }^{6}$ Fariba Ramezanali, ${ }^{7}$ Mohammad Chehrazi
}

\begin{abstract}
Introduction: Two of the most frequent procedures performed on infertile women are two-dimensional ultrasound (2DUS) and three-dimensional ultrasound (3DUS). Hysteroscopy is considered as the gold standard for evaluation of acquired endometrial lesions in infertile women; however, 3DUS is used as a noninvasive, less expensive, and reliable assessment method for evaluation of the intrauterine lesions in infertile women. We aimed to compare the diagnostic efficiency between 3DUS and hysteroscopy in the detection of lesions (polyps, submucous leiomyoma, and synechiae) in infertile women.
\end{abstract}

Materials and methods: In this prospective observational study, infertile women $(n=155)$ with indication of hysteroscopy were scheduled to undergo 3DUS prior to hysteroscopy from September 2010 to 2011 . Women with suspected congenital uterine anomalies were excluded. The sensitivity and specificity values of 3DUS were compared with those of hysteroscopy. Hysteroscopy was used as the gold standard for diagnosis of intrauterine lesions in infertile women.

Results: Of the 155 women, 50 were found to have an intracavitary abnormality, 36 had polyps, 12 had myomas, and 7 had synechiae on hysteroscopic findings. Examination with 3DUS in the diagnosis of intrauterine lesions reached an accuracy of $94 \%$, and 92.15 and $96.9 \%$ of sensitivity and specificity respectively. Positive predictive value (PPV) was $83.9 \%$, and a negative predictive value (NPV) was $91.3 \%(\mathrm{LR}+=10.75, \mathrm{LR}+=0.065)$.

\footnotetext{
${ }^{1}$ Head, ${ }^{2,4,5,7}$ Research Assistant, ${ }^{3}$ Radiologist, ${ }^{6}$ Assistant Professor and Gynecologist

1,2,4,5 Department of Reproductive Imaging, Reproductive Biomedicine Research Center, Royan Institute for Reproductive Biomedicine, Academic Center for Education, Culture and Research, Tehran, Islamic Republic of Iran

${ }^{3}$ Department of Radiology, Tehran University of Medical Sciences, Tehran, Islamic Republic of Iran

${ }^{6}$ Department of Endocrinology and Female Infertility, Reproductive Biomedicine Research Center, Royan Institute for Reproductive Biomedicine, Academic Center for Education, Culture and Research, Tehran, Islamic Republic of Iran

${ }^{7}$ Department of Epidemiology and Reproductive Health Reproductive Epidemiology Research Center, Royan Institute for Reproductive Biomedicine, Academic Center for Education Culture and Research, Tehran, Islamic Republic of Iran

Corresponding Author: Firoozeh Ahmadi, Head, Department of Reproductive Imaging, Reproductive Biomedicine Research Center Royan Institute for Reproductive Biomedicine Academic Center for Education, Culture and Research, Tehran Islamic Republic of Iran, Phone: +982123562254, e-mail: dr.ahmadi1390@ gmail.com
}

Conclusion: According to our results, 3DUS has a reliable diagnostic accuracy for intrauterine lesions, and it may limit unnecessary hysteroscopy in patients with normal results.

Keywords: Hysteroscopy, Three-dimensional sonography, Uterine cavity.

How to cite this article: Ahmadi F, Haghighi H, Ghahremani Z, Niknejad F, Akhbari F, Ramezanali F, Chehrazi M. Diagnostic Accuracy of Three-dimensional Ultrasonography in Detection of Endometrial Lesions compared with Hysteroscopy in Infertile Women. Donald School J Ultrasound Obstet Gynecol 2016;10(4):393-397.

Source of support: Nil

Conflict of interest: None

\section{INTRODUCTION}

Two-dimensional ultrasound (2DUS) and threedimensional ultrasound (3DUS) are routinely used as a core part in infertility evaluation. Prior to assisted reproductive technology (ART) cycles, suspected uterine cavity abnormalities can be detected by hysteroscopy. ${ }^{1}$ Hysteroscopy is also marked as the gold standard for the assessment of infertile women. ${ }^{2-4}$ However, 2DUS and later 3DUS are being applied increasingly to determine preoperatively the presence and extent of the surgical pathology. Also, 3DUS is initially regarded as an experimental investigation tool, which soon developed into a useful complement to 2DUS for evaluation of uterine pathology. In addition, 3DUS may offer a better ground for therapy or verification of results by providing more precise anatomical sections to explore the endometrial cavity, the relations of submocusal myomata and possible encroachment on the cavity, the endometrial polyp, as well as the extent of cavity involvement in intrauterine adhesions. Therefore, it may help to reserve the hysteroscopy for uterine lesions that could benefit from this treatment.

In this study, we aimed to emphasize the diagnostic capabilities of 3DUS with regard to intralesions detection (polyps, submucous leiomyoma, and synechiae) as compared with a gold standard (hysteroscopy).

\section{MATERIALS AND METHODS}

This prospective observational study included a group of infertile women $(n=155)$ with indication of hysteroscopy 
Table 1: Characteristic of all patients in the study

\begin{tabular}{ll}
\hline Characteristic & Values $(n=153)$ \\
\hline Mean age & $34 / 4$ SD \\
Type of infertility & \\
$\quad$ Primary infertility & 116 \\
$\quad$ Secondary infertility & 37 \\
Duration of infertility (years) & $7.3 \mathrm{SD}$ \\
Indication of hysteroscopy & \\
$\quad$ Suspected intrauterine lesions & 72 \\
$\quad$ Unexplained infertility & 11 \\
$\quad$ Repeated spontaneous abortion & 98 \\
$\quad$ Failure following in vitro fertilization & 42 \\
\hline SD: Standard deviation &
\end{tabular}

(Table 1) who were scheduled to undergo 3DUS prior to hysteroscopy at Royan Institute from September 2010 to 2011. Exclusion criteria were suspected congenital uterine anomalies. Both hysteroscopy and 3DUS examinations were performed on the same day (with no time interval), 7 to 10 days from the start of menstruation.

Hysteroscopy has been used as the gold standard because its value was previously confirmed in studies.$^{2-4}$ In this study, 3DUS images were interpreted only by an experienced radiologist with a special training in gynecology [7 years' experience with three-dimensional extended imaging (3DXI)], whereas hysteroscopy was performed by an experienced gynecologist. Our surgeon was also blind to ultrasound findings. We used 3DXI (ACCUVIX XQ, Medison, South Korea) ultrasound with a 6.5-MHz transvaginal probe.

After a baseline transabdominal and transvaginal 2DUS, analysis of endometrial cavity was completed using multiplaner 3D and multislice view. Representative transverse and longitudinal images were then obtained, while 3DXI was employed as a new display modality. Furthermore, this study was approved by the Research Ethics Committee and the Institutional Review Board of Royan Institute, and informed written consent was obtained from the patients.

Diagnosis by 3DUS was recorded as follows: (1) Endometrial polyp (hyperechoic thickening of the endometrial mucosa) (Fig. 1), (2) intrauterine adhesions (irregularities of endometrium) (Fig. 2), and (3) submucous leiomyoma (a solid, whorled, and mixed echogenic tumor disrupting and affecting the endometrial interfaces) (Figs $3 \mathrm{~A}$ and $\mathrm{B})$. In this study, size and location of polyps and submucous leiomyoma were documented, indicating the display and the outline of the region-of-interest (ROI) in three perpendicular planes, simultaneously.

The hysteroscopy was performed under either general or regional anesthesia using a $4 \mathrm{~mm}$ hysteroscope system (Karl Storz-GmbH \& Co., Tuttlingen, Germany) by an expert gynecologist.

\section{Statistical Method}

Accuracy was represented using the following values: Sensitivity, specificity, positive predictive value (PPV) and negative predictive value (NPV), positive and negative likelihood ratios, and overall accuracy. Precision of these terms was shown using confidence interval. A probability

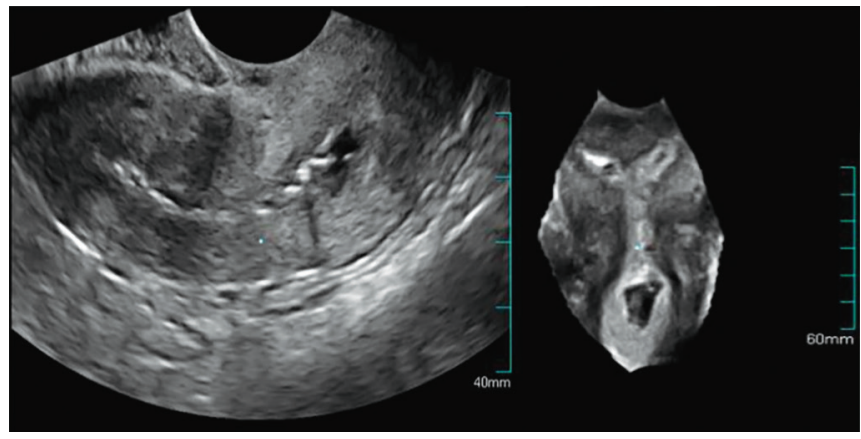

Fig. 2: Sagittal and coronal view: The exact location and the extent of uterine synechiae is more specified in coronal view

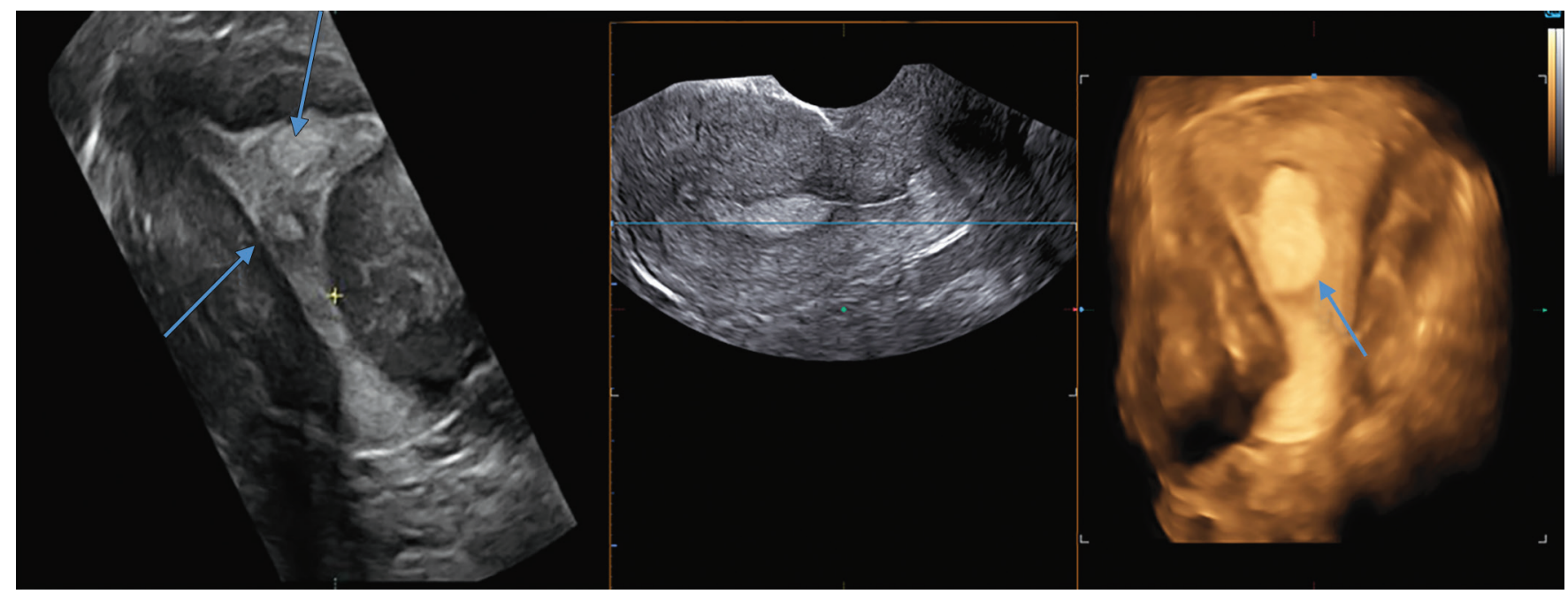

Fig. 1: Endometrial polyps (arrows) on 3DUS 

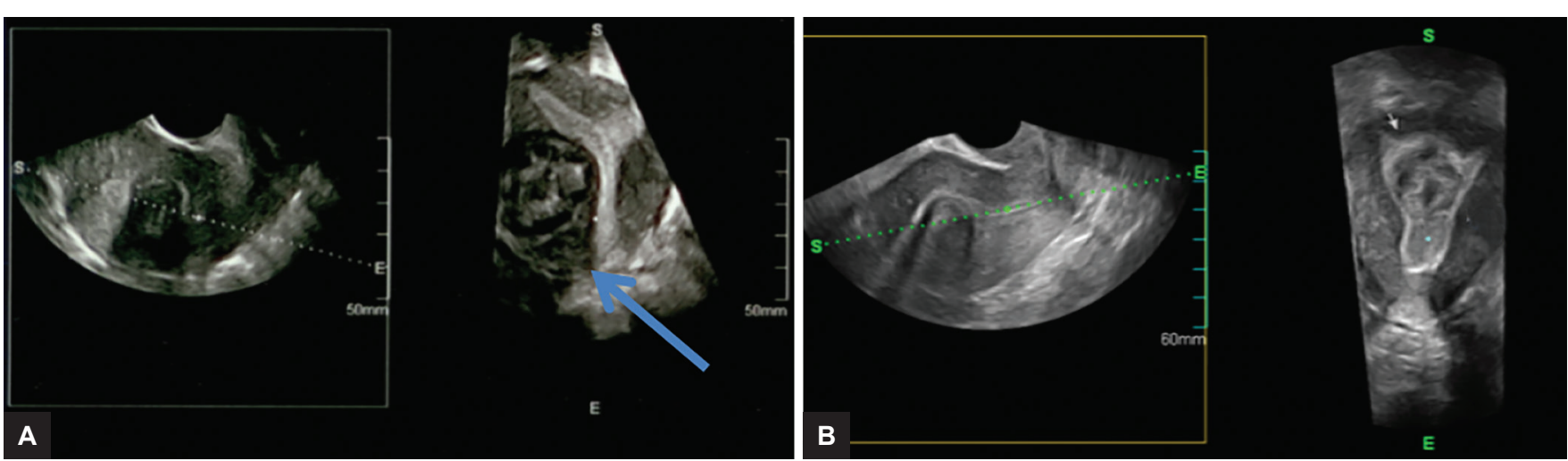

Figs 3A and B: Sagittal and coronal view show submucosal leiomyoma (arrow) causes displacement and distortion of the uterine cavity

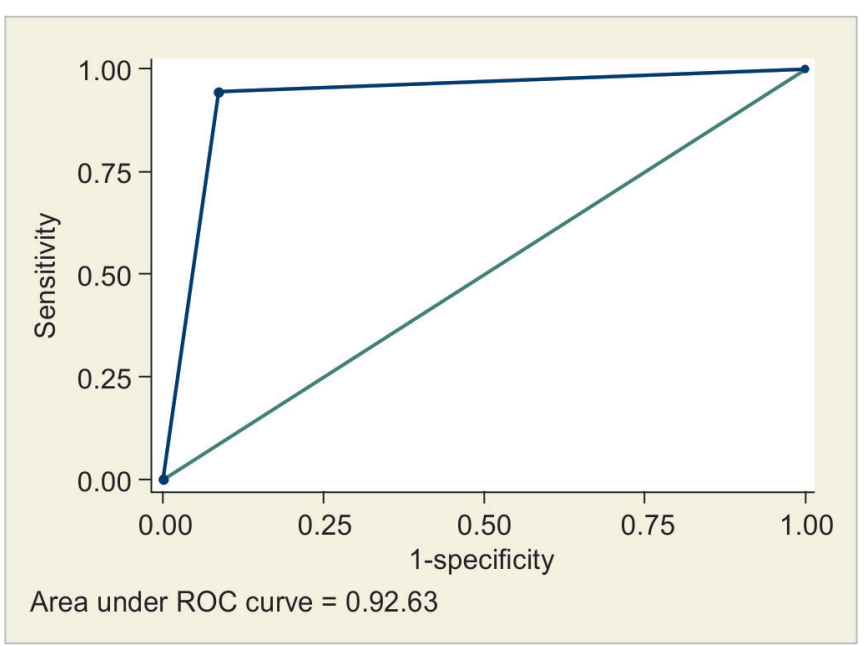

Graph 1: Accuracy of test according the area under the ROC curve

value ( $\mathrm{p}$-value) less than 0.05 was considered significant. Also, receiver operating characteristic (ROC) curve was used to depict area under curve. The ROC curve graph included sensitivity $v s$ one minus specificity (Graph 1$)$. Data were analyzed using Stata version 11 (StataCorp LP, College Station, TX, USA).

\section{RESULTS}

This study involved 3DUS examination in 155 infertile women prior to undergoing hysteroscopy. Two patients were excluded due to cancellation of hysteroscopy. The mean age of study group was $34.4 \pm 3.83 \mathrm{SD}(20-48)$ years (Table 1).

The duration of couples' infertility ranged from 1 to 30 $($ mean $=7.37)$ years. For the calculation of diagnostic accuracy, all patients (153) were examined by both the diagnostic test (3DUS) and the gold standard test (hysteroscopy). Of the 153 women, 56 (35.6\%) had abnormal test results
Table 2: Detection of intracavitary uterine lesions: Comparison of results of hysteroscopy to 3DUS

\begin{tabular}{llll}
\hline & \multicolumn{2}{c}{ Hysteroscopy } & \\
\cline { 2 - 3 } 3DUS & Abnormal & Normal & Total \\
\hline Abnormal & 47 & 9 & 56 \\
Normal & 3 & 94 & 97 \\
\hline Total & 50 & 103 & 153 \\
\hline
\end{tabular}

(at least one intrauterine lesion) and 97 (63\%) had normal test results on 3DUS. Graph 1 shows ROC curve, including sensitivity vs one minus specificity. Normal and abnormal results of intrauterine lesions detected by hysteroscopy and 3DUS are illustrated in Table 2. As shown in Table 3 , the 3DUS had $92.15 \%$ accuracy, $94 \%$ sensitivity, $91.3 \%$ specificity, $83.9 \%$ PPVs, and $96.9 \%$ NPVs for the diagnosis of intrauterine lesions as compared with the gold standard.

The hysteroscopy detected 50 cases of uterine abnormalities, while 3DUS was in complete agreement in 47 of 50 cases. The results also showed that 4 patients had more than one intrauterine lesion. The hysteroscopy findings revealed 36 polyps, 12 leiomyoma, and 7 synechiae, while 3 DUS findings were in complete agreement in $23 / 36$ cases of polyps and $8 / 12$ cases of submucosal leiomyoma.

\section{DISCUSSION}

The acquired uterine anomalies are important causes of female infertility, hence investigation of the uterine cavity plays an integral role in the infertility evaluation, particularly prior to ART cycles. ${ }^{5}$ The noninvasive ultrasound imaging modalities, such as 2DUS, 3DUS, and three-dimensional hysterosonography $(3 \mathrm{DHS})^{6}$ are useful tools to improve the effective treatment and management of infertility.

Recently, 3DXI has provided the ability to obtain simultaneous display of multiple sequential of acquired

Table 3: Diagnostic accuracy of three-dimensional ultrasound vs hysteroscopy in the diagnosis of intrauterine lesions

\begin{tabular}{lllllllll}
\hline & Accuracy & Sensitivity & Specificity & PPV & NPV & $L R^{+} . L R^{-}$ & AUC & $k a p p a$ \\
\hline 3DUS & $92.1 \%$ & $94 \%$ & $91.3 \%$ & $83.9 \%$ & $96.9 \%$ & LR $^{+}=10.75 . \mathrm{LR}^{-}=0.065$ & $91.6(\mathrm{Cl}=85.9-95.4)$ & 82.7 \\
\hline
\end{tabular}


volume scans in A, B, and C planes. ${ }^{7}$ Although the simultaneous display of three planes in 3DUS provides visualization of the anatomy in planes, the procedure is unable to provide the direct scanning. The reconstructed planes are very useful in the evaluation of anomalies ${ }^{8-10}$ and also of utmost importance for reliable investigation of intrauterine lesions by offering more precise analysis of spatial relationships among structures under study. Furthermore, 3DUS provides a more accurate visualization of the adhesions and extent of cavity destruction, ${ }^{11}$ better determination of the degree of intramural involvement of leiomyoma, and the exact location and size of polyps. ${ }^{9}$ This preoperative information reduces complication rates and duration of operation.

Magnetic resonance imaging is also an effective secondary imaging modality when more information is needed on the location and architecture of a fibroid, especially when they are large and multiple.

The benefit and even the superiority of 3DUS over commonly 2DUS are its ability to acquire coronal view of the uterus as well as offline assessment, which reduces the duration of examination and causes less discomfort for the patients. ${ }^{12}$

The efficiency of 3DUS has been confirmed by three individual studies conducted by Ali, ${ }^{13}$ Bocca, ${ }^{14}$ and Zvâncă. ${ }^{15}$ Diagnostic accuracy of 3DUS was also investigated among infertile, premenopausal, and postmenopausal women with abnormal uterine bleeding (AUB) who mostly suffered from intrauterine lesions. ${ }^{13-15}$ The number of studies comparing diagnostic accuracy between 3DUS and hysteroscopy are few. To consider the obtained data, these studies concluded that 3DUS is an accurate method in detection of endometrial cavity abnormalities with sensitivity and specificity values of more than 63 and $80 \%$ respectively. ${ }^{16,17}$ In this study, the sensitivity and specificity values of 3DUS and the related values of hysteroscopy are within the expected range as compared with previous study demonstrating a sensitivity and specificity values of 93.75 and $81.25 \%$ respectively, in patients with AUB. ${ }^{17}$

In spite of many advantages, 3DUS may not provide qualitative image when the endometrium is thin. Additionally, this technique is not easily available and confined to specialized centers.

\section{CONCLUSION}

According to our results, 3DUS has a reliable diagnostic accuracy for intrauterine lesions, and it may limit unnecessary hysteroscopy in patients with normal results.

\section{ACKNOWLEDGMENT}

Authors would like to thank the staff of imaging department for their assistance in figure preparation. All the authors participated sufficiently in the work to take public responsibility for the content. There is no financial interest that might inappropriately influence or interfere with research findings, and there is no relevant financial or nonfinancial relationship in the products or services described, reviewed, evaluated, or compared in this presentation.

\section{REFERENCES}

1. Shalev J, Meizner I, Bar-Hava I, Dicker D, Mashiach R, Ben-Rafael Z. Predictive value of transvaginal sonography performed before routine diagnostic hysteroscopy for evaluation of infertility. Fertil Steril 2000 Feb;73(2):412-417.

2. Loverro G, Nappi L, Vicino M, Carriero C, Vimercati A, Selvaggi L. Uterine cavity assessment in infertile women: comparison of transvaginal sonography and hysteroscopy. Eur J Obstet Gynecol Reprod Biol 2001 Dec;100(1):67-71.

3. Sylvestre C, Child TJ, Tulandi T, Tan SL. A prospective study to evaluate the efficacy of two-and three-dimensional sonohysterography in women with intrauterine lesions. Fertil Steril 2003 May;79(5):1222-1225.

4. Brown SE, Coddington CC, Schnorr J, Toner JP, Gibbons W, Oehninger S. Evaluation of outpatient hysteroscopy, saline infusion hysterosonography, and hysterosalpingography in infertile women: a prospective, randomized study. Fertil Steril 2000 Nov;74(5):1029-1034.

5. Pundir J, El Toukhy T. Uterine cavity assessment prior to IVF. Women's Health 2010 Nov;6(6):841-848.

6. Ahmadi F, Rashidy Z, Haghighi H, Akhoond M, Niknejadi M, Hemat M, Shamsipour. Uterine cavity assessment in infertile women: sensitivity and specificity of three-dimensional hysterosonography versus hysteroscopy. Iran J Reprod Med 2013;11(12):977-982.

7. Zafarani F, Ahmadi F. Evaluation of intrauterine structural pathology by three-dimensional sonohysterography using an extended imaging method. Int J Fertil Steril 2013 Apr;7(1): $1-6$.

8. Moini A, Mohammadi S, Hosseini R, Eslami B, Ahmadi F. Accuracy of 3-dimensional sonography for diagnosis and classification of congenital uterine anomalies. J Ultrasound Med 2013 Jun;32(6):923-927.

9. Ahmadi F, Zafarani F, Niknejadi M, Haghiahi H, Vosough Taqi Dizaj A. Application of 3D ultrasonography in detection of uterine abnormalities. Int J Fertil Steril 2011 Jan;4(4):144-147.

10. Ahmadi F, Haghighi H. Detection of congenital mullerian anomalies by real-time 3D sonography. J Reprod Infertil 2012 Jan;13(1):65-66.

11. Knopman J, Copperman AB. Value of 3D ultrasound in the management of suspected Asherman's syndrome. J Reprod Med 2007 Nov;52(11):1016-1022.

12. Tan I, Robertson M. The role of imaging in the investigation of Asherman's syndrome. Aust J Ultrsound Med 2011 Aug;14(3):15-18.

13. Ali AFM, Hamed WM, Shalabi HS, Abdel Rahman AE. Threedimensional ultrasound as a single step for evaluation of infertile women. Fertil Steril 2002 Sep;78:S93.

14. Bocca S, Ragulin E, Stadtmauer L, Abuhamad A, Oehninger S. Three dimensional ultrasound (3D US): high accuracy, low cost and minimal discomfort in the diagnosis of uterine anomalies. Fertil Steril 2007 Sep;88:S203. 
15. Zvâncă M, Vlădăreanu R, Kurjak A, Al Bahar AJ. Conventional versus $3 \mathrm{D}$ ultrasound for the investigation of infertile women. Iran J Reprod Med 2008;6(3):109-117.

16. Haemila FA, Youssef D, Hassan M, Soliman A, Mossad M. A prospective comparative study of 3-D ultrasonography and hysteroscopy in detecting uterine lesions in premeno- pausal bleeding. Middle East Fertility Soc J 2005 Dec;10(3): 238-243.

17. Hosny I, Elghawabit H, Mosaad M. The role of 2D, 3D ultrasound and color Doppler in the diagnosis of benign and malignant endometrial lesions. J Egypt Natl Canc Inst 2007 Dec;19(4):275-281. 